\title{
Approximation of Stochastic Programming Problems
}

\author{
Christine Choirat ${ }^{1}$, Christian Hess $^{2}$, and Raffaello Seri ${ }^{3}$
}

1 Dipartimento di Economia, Università degli Studi dell'Insubria, Via Ravasi 2, 21100 Varese, Italy, cchoirat@eco.uninsubria.it,

2 Centre de Recherche Viabilité, Jeux, Contrôle, Université Paris 9 Dauphine, 75775 Paris CEDEX, France, Christian.Hess@dauphine.fr

3 Dipartimento di Economia, Università degli Studi dell'Insubria, Via Ravasi 2, 21100 Varese, Italy, rseri@eco.uninsubria.it

Summary. In Stochastic Programming, the aim is often the optimization of a criterion function that can be written as an integral or mean functional with respect to a probability measure $\mathbb{P}$. When this functional cannot be computed in closed form, it is customary to approximate it through an empirical mean functional based on a random Monte Carlo sample. Several improved methods have been proposed, using quasi-Monte Carlo samples, quadrature rules, etc. In this paper, we propose a result on the epigraphical approximation of an integral functional through an approximate one. This result allows us to deal with Monte Carlo, quasi-Monte Carlo and quadrature methods. We propose an application to the epi-convergence of stochastic programs approximated through the empirical measure based on an asymptotically mean stationary (ams) sequence. Because of the large scope of applications of ams measures in Applied Probability, this result turns out to be relevant for approximation of stochastic programs through real data.

\section{Introduction}

In Stochastic Programming, one has often to solve an optimization problem of the form (1) (see [4], p. 332):

$$
\inf _{x \in \mathbf{X}} \mathbb{E} g(Y, x)
$$

where $\mathbf{X} \subset \mathbb{R}^{p}$ and $Y$ is a $\mathbb{R}^{q}$-valued random variable. Most of the time the integral functional (also called the mean functional) $\mathbb{E} g(Y, x)$ cannot be explicitly calculated, but can be approximated through sampling methods (see [4], Chapter 10): ${ }^{4}$ suppose that a sample of realizations of the random variable

\footnotetext{
${ }^{4}$ Much more general stochastic programming problems can be dealt with in this way, such as multistage stochastic programs with recourse and stochastic programs with chance constraints.
} 
$Y$, say $\left(Y_{i}\right)_{i=1, \ldots, n}$, are available. We would like to find conditions under which the solution of the approximated problem

$$
\inf _{x \in \mathbf{X}} \frac{1}{n} \sum_{i=1}^{n} g\left(Y_{i}, x\right)
$$

converges almost surely to the solution of the original problem (1). It is common to assume that $\left(Y_{i}\right)_{i=1, \ldots, n}$ is a sample of independent and identically distributed realizations of the random variable $Y$ and that the approximated objective function converges uniformly on $\mathbf{X}$ almost surely to the original one:

$$
\sup _{x \in \mathbf{X}}\left|\frac{1}{n} \sum_{i=1}^{n} g\left(Y_{i}, x\right)-\mathbb{E} g(Y, x)\right| \rightarrow 0 \quad \text { as; }
$$

this can be cast in the framework of the Glivenko-Cantelli problem and implies the convergence of the minimizers.

The previous structure can be extended to encompass also the so-called $M$-estimation used in Statistics and Econometrics, that is, estimation obtained by optimizing a function with respect to some parameters.

In the present paper, we shall focus our attention on epigraphical convergence for sequences of stochastic functions defined on a metric space. Epigraphical convergence (epi-convergence, for short) is weaker than uniform convergence, but it is well-suited to approximate minimization problems. Indeed, under suitable compactness assumptions, it entails the convergence of infima and minimizers (see e.g. [3] or [9]). A symmetric notion, called hypographical convergence, enjoys similar properties with respect to maximization problems. Further, as it is known, this type of convergence is closely related to the Painlevé-Kuratowski convergence for sequences of subsets, so that it has an interesting geometric interpretation allowing for connections with the theory of random sets.

Similar results have been already derived in [2], when $\left(Y_{i}\right)_{i=1, \ldots, n}$ is a sample of independent and identically distributed Monte Carlo realizations, in $[14,15]$, when $\left(Y_{i}\right)_{i=1, \ldots, n}$ is a sample of pairwise independent and identically distributed Monte Carlo realizations, and in $[32,19,33,7]$, when $\left(Y_{i}\right)_{i=1 \ldots, n}$ is a stationary ergodic sequence. A particular mention should be made to [27], where a general result similar in scope to the present one is proposed.

The next Section contains some preliminaries on epi-convergence (other results on epi-convergence are gathered in Appendix A). Then, Section 3, and Theorem 1 in particular, provides a result on the epi-convergence of the objective functions to be minimized. This result is motivated by examples. Section 4 studies in more details its application to asymptotically mean stationary processes. The proofs are deferred to Section 5 . 


\section{Preliminaries on Epi-convergence}

In this Section, we define the concept of epi-convergence. Its main properties are briefly recalled in Appendix A. For a more complete treatment of the subject, we refer the reader to the monographs [3] or [9].

Let $(E, d)$ be a metric space and $\phi: E \rightarrow \overrightarrow{\mathbb{R}}=[-\infty,+\infty]$ be a function from $X$ into the extended reals. Its epigraph (or upper graph) is defined by:

$$
\operatorname{Epi}(\phi)=\{(x, \lambda) \in E \times \mathbb{R}: \phi(x) \leq \lambda\} .
$$

Its hypograph (or lower graph) Hypo $(\phi)$ is defined by reversing the inequality. Further, given a sequence $\left(\phi_{n}\right)_{n>1}$ of functions from $E$ into $\overline{\mathbb{R}}$, the functions epi $-\lim \inf \phi_{n}$ and epi $-\lim \sup \phi_{n}$ are defined on $E$ by

$$
\begin{aligned}
\operatorname{epi}-\liminf \phi_{n}(x) & =\sup _{k \geq 1} \liminf _{n \rightarrow \infty} \inf _{y \in \mathrm{B}(x, 1 / k)} \phi_{n}(y), \\
\text { epi }-\lim \sup \phi_{n}(x) & =\sup _{k \geq 1} \limsup _{n \rightarrow \infty} \inf _{y \in \mathrm{B}(x, 1 / k)} \phi_{n}(y),
\end{aligned}
$$

where $\mathrm{B}(x, 1 / k)$ denotes the open ball of radius $1 / k$ centered at $x$. These functions are called respectively the lower epi-limit and the upper epi-limit of $\left(\phi_{n}\right)_{n \geq 1}$. Moreover, both functions are lower semi-continuous (Isc for short) and one has for all $x \in E$

$$
\text { epi }-\liminf \phi_{n}(x) \leq \operatorname{epi}-\lim \sup \phi_{n}(x) .
$$

When the equality holds in (4) at some point $x \in E$, the sequence $\left(\phi_{n}\right)$ is said to be epi-convergent at $x$. If this holds for all $x \in E$, the common value defines a function $\phi$ that is called the epi-limit of the sequence. This is denoted by $\phi=$ epi- $\lim \phi_{n}$.

Equalities (2) and (3) have a geometric counterpart involving the PainlevéKuratowski convergence of epigraphs on the space of closed sets of $E \times \mathbb{R}$. Let us give a general definition. Given a sequence of sets $\left(C_{n}\right)_{n \geq 1}$ in the metric space $E$, we define

$$
\begin{gathered}
\mathrm{PK}-\liminf _{\mathrm{n} \rightarrow \infty} C_{n} \triangleq\left\{x \in E: x=\lim x_{n}, x_{n} \in C_{n}, \forall n \geq 1\right\} \\
\mathrm{PK}-\limsup _{\mathrm{n} \rightarrow \infty} C_{n} \triangleq\left\{x \in E: x=\lim x_{i}, x_{i} \in C_{n(i)}, \forall i \geq 1\right\}
\end{gathered}
$$

where $\left(C_{n(i)}\right)_{i \geq 1}$ is a subsequence of $\left(C_{n}\right)_{n \geq 1}$. The subsets $\mathrm{PK}-\lim \inf C_{n}$ and $\mathrm{PK}-\lim \sup C_{n}$ are the lower limit and the upper limit of the sequence $\left(C_{n}\right)_{n>1}$. They are both closed and satisfy $\mathrm{PK}-\lim \inf C_{n} \subset \mathrm{PK}-\lim \sup C_{n}$. A sequence $\left(C_{n}\right)_{n \geq 1}$ is said to converge to $C$ in the sense of Painlevé-

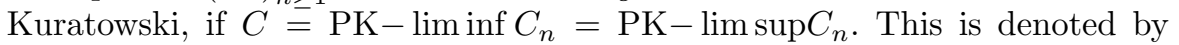
$C=\mathrm{PK}-\lim _{n \rightarrow \infty} C_{n}$. As mentioned above, this notion is strongly connected with epi-convergence: the sequence of functions $\phi_{n}$ epi-converges to $\phi$ on $E$ if and only if the sequence $\left(\operatorname{Epi}\left(\phi_{n}\right)\right)_{n \geq 1} \mathrm{PK}$-converges to Epi $(\phi)$, in $E \times \mathbb{R}$. 


\section{Main Results}

In this Section, we present the mathematical framework of our main result.

Consider a metric space $(\mathbf{X}, d)$ (usually a subset of an Euclidean space $\mathbb{R}^{q}$ ) endowed with its Borel $\sigma$-algebra $\mathcal{B}(\mathbf{X})$ and a metric space $(\mathbf{Y}, \rho)$ endowed with its Borel $\sigma$-algebra $\mathcal{B}(\mathbf{Y})$ and with a probability measure $\mathbb{P}$ defined on $(\mathbf{Y}, \mathcal{B}(\mathbf{Y}))$. We want to approximate the stochastic programming problem:

$$
\inf _{x \in \mathbf{X}} \mathbb{E} g(Y, x)
$$

where $\mathbb{E}$ is the mean with respect to $\mathbb{P}$, through a simpler problem given by

$$
\inf _{x \in \mathbf{X}} \mathbb{E}_{n} g(Y, x)
$$

where $\mathbb{E}_{n}$ stands for the expectation under $\mathbb{P}_{n} . \mathbb{P}_{n}$ will often be a transition probability measure, i.e. a probability measure depending on a random parameter $\omega$; this point will be made clearer in the following. We look for some conditions under which the solution of the approximated problem (6) converges to the solution of (5).

Some remarks and definitions about $g, \mathbf{Y}$ and $\mathbb{P}_{n}$ are necessary. We say that an extended function $g: \mathbf{Y} \times \mathbf{X} \rightarrow \overline{\mathbb{R}}$ is an integrand if it is $\mathcal{B}(\mathbf{Y}) \otimes$ $\mathcal{B}(\mathbf{X})$ - measurable. ${ }^{5}$ Furthermore, $g$ is called a normal integrand if $g(y, \cdot)$ is a lower semi-continuous (Isc) function for $\mathbb{P}$-almost all $y \in \mathbf{Y}$. The function $g$ is said to be $k$-Lipschitz on $\mathbf{X}$ if for $\mathbb{P}$-almost all $y \in \mathbf{Y}$ and for all $x, x^{\prime} \in \mathbf{X}$,

$$
\left|g(y, x)-g\left(y, x^{\prime}\right)\right| \leq k \cdot d\left(x, x^{\prime}\right) .
$$

An integrand is said to be positive if, for $\mathbb{P}$-almost every $y \in \mathbf{Y}, g(y, \cdot)$ takes on its values in $[0,+\infty]$. Given a normal integrand $g$ and an integer $k \geq 1$, the Lipschitz approximation of order $k$ of $g$ is defined by:

$$
g^{k}(y, x) \triangleq \inf _{x^{\prime} \in \mathbf{X}}\left\{g\left(y, x^{\prime}\right)+k \cdot d\left(x, x^{\prime}\right)\right\}, \quad k \geq 1 .
$$

We will resort to Lipschitz approximations to derive a characterization of epi-convergence (see Proposition 5) used in our main result.

As concerns $\mathbf{Y}$, since our aim is to approximate the mean $\mathbb{E} g(Y, x)=$ $\int_{\mathbf{Y}} g(y, x) \mathbb{P}(\mathrm{d} y)$ through $\mathbb{E}_{n} g(Y, x)=\int_{\mathbf{Y}} g(y, x) \mathbb{P}_{n}(\mathrm{~d} y)$, the only constraint is the kind of space on which the approximate probability measure $\mathbb{P}_{n}$ can be defined: as an example, if the space $\mathbf{Y}$ is the unit hypercube $[0,1]^{q}$, we can take $\mathbb{P}_{n}$ to be the empirical measure defined by a quasi-Monte Carlo sequence (see below). On the other hand, Monte Carlo can be used also in more abstract spaces.

On the other hand, also the nature of the probability measure $\mathbb{P}_{n}$ has to be better specified.

\footnotetext{
${ }^{5}$ For real-valued functions, this corresponds exactly to the definition of a measurable random real function in [22] (Definition III-4-4, p. 86) and [12] (Definition 1, p. 157).
} 
Example 1. Consider the case of Monte Carlo integration through a sample of independent and identically distributed (iid) random variables $\left(Y_{i}\right)_{i=1, \ldots, n} . \mathbb{P}_{n}$ can be specified by the empirical distribution:

$$
\mathbb{P}_{n}(\omega, A) \triangleq \frac{1}{n} \sum_{i=1}^{n} \delta_{Y_{i}(\omega)}(A)
$$

for a set $A$, where $\delta_{y}(\cdot)$ is a Dirac delta. Remark that the distribution $\mathbb{P}_{n}$ is random (and depends on $\omega$ ) since the sequence of iid random variables $\left(Y_{i}\right)_{i=1, \ldots, n}$ defining $\mathbb{P}_{n}$ is random.

This situation is quite general. Therefore, we suppose that $\mathbb{P}_{n}$ takes the following form:

$$
\mathbb{P}_{n}(\omega, A)=\sum_{i=1}^{n} w^{i} \cdot \delta_{Y_{i}(\omega)}(A)
$$

where $\left(w^{i}\right)_{i=1, \ldots, n}$ is a sequence of weights and the points $\left(Y_{i}(\omega)\right)_{i=1, \ldots, n}$ can be chosen in a stochastic or deterministic way. In a rigorous probabilistic setting, $\mathbb{P}_{n}$ is defined as a transition probability (see e.g. [22], Chapter III-2), i.e. as the mapping:

$$
\begin{aligned}
\mathbb{P}_{n}: \Omega \times \mathcal{B}(\mathbf{Y}) & \rightarrow \quad[0,1] \\
(\omega, A) & \mapsto \mathbb{P}_{n}(\omega, A)
\end{aligned}
$$

such that:

- for any $\omega \in \Omega, \mathbb{P}_{n}(\omega, \cdot)$ is a probability on $(\mathbf{Y}, \mathcal{B}(\mathbf{Y}))$;

- for any $A \in \mathcal{B}(\mathbf{Y}), \mathbb{P}_{n}(\cdot, A)$ is $\mathcal{A}$-measurable.

In order to stress the characteristics of $\mathbb{P}$ and $\mathbb{P}_{n}$, we will use the notations $\mathbb{E} g(Y, x) \triangleq \int_{\mathbf{Y}} g(y, x) \mathbb{P}(\mathrm{d} y)$ and $\left[\mathbb{E}_{n} h(Y, x)\right](\omega) \triangleq \int_{\mathbf{Y}} h(y, x) \mathbb{P}_{n}(\omega, \mathrm{d} y)$ to indicate respectively the mean with respect to $\mathbb{P}$ and $\mathbb{P}_{n}$.

Example 2. (Monte Carlo) The kind of approximation introduced in Example 1 is considered e.g. in [2]. Hess $[14,15]$ covers the case in which $\left(Y_{i}\right)_{i=1, \ldots, n}$ is a sample of identically distributed and pairwise independent random variables. The situation in which $\left(Y_{i}\right)_{i=1, \ldots, n}$ is a stationary ergodic sequence is considered in $[32,19,33,7]$.

Example 3. (Quasi-Monte Carlo) A different method is to use quasi-Monte Carlo integration methods: in this case, the random sample is substituted by a low discrepancy point set $\left(y_{i}\right)_{i=1, \ldots, n}$ and $\mathbb{P}_{n}$ is given by $\mathbb{P}_{n}(A) \triangleq$ $\frac{1}{n} \sum_{i=1}^{n} \delta_{y_{i}}(A)$ for $A \in \mathcal{B}(\mathbf{Y})$; remark that in this case the empirical distribution is not random. Quasi-Monte Carlo integration methods are in general more efficient than Monte Carlo ones. However, most of quasi-Monte Carlo methods are limited to sequences of points uniformly scattered in the unit hypercube $[0,1]^{d}$ (see [10], Section 1.5, for some algorithms yielding uniform 
points on sets different from the unit hypercube). Using an argument based on the probability integral transform (see e.g. [1], for the result and [27], for applications to Stochastic Programming) and on its multivariate generalization ([29]; see [20], p. 354, for an application to Numerical Analysis), it is possible to create random vectors with arbitrary distributions: however, this method often requires the evaluation of complex functions (quantile functions of conditional distributions) and can be quite time-demanding in practice. Moreover, quasi-Monte Carlo methods require more stringent hypotheses on the behavior of the function $g$ : indeed, while any Lebesgue integrable function can be integrated using Monte Carlo algorithms, low discrepancy point sets require the function to be Riemann integrable (however, in order to derive a convergence rate it also has to be of bounded variation in the sense of HardyKrause). Also hybrid techniques in which a deterministic sequence is perturbed by stochastic mechanisms can be considered, such as the randomized nets of Owen $([24,23])$ : in [18], the author applies randomized quasi-Monte Carlo methods to stochastic programming.

Example 4. (Quadrature Rules) Faster convergence rates can be obtained using quadrature rules. In this case, the approximating measure is given by:

$$
\mathbb{P}_{n}(A)=\sum_{i=1}^{n} w^{i, n} \cdot \delta_{y_{i, n}}(A)
$$

for $A \in \mathcal{B}(\mathbf{Y})$, where the sequence of nodes $\left(y_{i, n}\right)_{i=1, \ldots, n}$ and the sequence of weights $\left(w^{i, n}\right)_{i=1, \ldots, n}$ (often constrained to respect $\sum_{i=1}^{n} w^{i, n}=1$ ) are choosen so to optimize a certain measure of accuracy. As an example, $n$-point Gaussian quadrature is obtained imposing that the integrals with respect to $\mathbb{P}$ and $\mathbb{P}_{n}$ coincide for all polynomials of degree $2 n-1$ or less. The use of these methods for the approximation of stochastic programming problems has been proposed in $[26,27,25]$.

Now, we come to our main result on the epi-convergence of approximate Stochastic Programming problems. We start introducing some hypotheses that will be useful in the following.

Hypothesis 1: $g$ is a normal integrand on $\mathbf{Y} \times \mathbf{X}$ with values in $\overline{\mathbb{R}}_{+}$. Moreover, the function $\mathbb{E} g(Y, \cdot)$ is not identically $+\infty$ on $\mathbf{X}$.

Hypothesis 2: $\mathbb{P}$ is a probability measure defined on the measurable space $(\mathbf{Y}, \mathcal{B}(\mathbf{Y})) .\left(\mathbb{P}_{n}\right)_{n}$ is a sequence of transition probabilities defined by $(7)$ for each $n \geq 1$.

Hypothesis 3: For any $k \in \mathbb{N}^{*}$ and any $x \in \mathbf{X}^{\star}$ (where $\mathbf{X}^{\star}$ is a dense subset of $\mathbf{X})$ :

$$
\liminf _{n \rightarrow \infty}\left[\mathbb{E}_{n}\left(g^{k}(Y, x)\right)\right](\omega)=\mathbb{E} g^{k}(Y, x), \quad \forall \omega \in \Omega \backslash N_{1}(x, k),
$$

where $N_{1}(x, k)$ is a negligible set. 
Hypothesis 4: For any $x \in \mathbf{X}^{\star}$ :

$$
\limsup _{n \rightarrow \infty}\left[\mathbb{E}_{n}(g(Y, x))\right](\omega)=\mathbb{E} g(Y, x), \quad \forall \omega \in \Omega \backslash N_{2}(x),
$$

where $N_{2}(x)$ is a negligible set.

Theorem 1. Let $(\mathbf{X}, d)$ be a metrizable separable space endowed with its Borel $\sigma$-algebra $\mathcal{B}(\mathbf{X}),(\mathbf{Y}, \rho)$ a metric space endowed with its Borel $\sigma$-algebra $\mathcal{B}(\mathbf{Y})$ and $g$ be a normal integrand on $\mathbf{Y} \times \mathbf{X}$ with values in $\overline{\mathbb{R}}$ respecting Hypothesis 1. Then, if Hypotheses 2, 3 and 4 hold, there exists a negligible subset $N$ of $\Omega$ such that, for every $x \in \mathbf{X}$ and $\omega \in \Omega \backslash N$, one has:

$$
\mathbb{E} g(Y, x)=\text { epi- } \lim _{n \rightarrow \infty}\left[\mathbb{E}_{n} g(Y, x)\right](\omega) .
$$

Remark 1. Hypothesis 1 can be replaced by one the following two hypotheses:

Hypothesis 1': $g$ is a normal integrand on $\mathbf{Y} \times \mathbf{X}$ with values in $\overline{\mathbb{R}}$ such that there exist a $\mathbb{P}$-integrable function $\beta(\cdot)$, a constant $a>0$ and $x_{0} \in \mathbf{X}$ such that, for $\mathbb{P}$-almost any $y \in \mathbf{Y}$ and any $x \in \mathbf{X}, g(y, x)+\beta(y)+a \cdot d\left(x, x_{0}\right) \geq$ 0 . Moreover, the function $\mathbb{E} g(Y, \cdot)$ is not identically $+\infty$ on $\mathbf{X}$.

Hypothesis 1": $g$ is a normal integrand on $\mathbf{Y} \times \mathbf{X}$ with values in $\overline{\mathbb{R}}$ such that for any $x \in \mathbf{X}$ there exists a neighborhood $W$ of $x$ and a $\mathbb{P}$-integrable function $\beta(\cdot)$ such that, for $\mathbb{P}$-almost any $y \in \mathbf{Y}$ and any $x \in W, g(y, x) \geq \beta(y)$. Moreover, the function $\mathbb{E} g(Y, \cdot)$ is not identically $+\infty$ on $\mathbf{X}$.

The main problem with the previous results is to show that Hypotheses 3 and 4 hold true.

Hypothesis 4 is usually much simpler to prove. Indeed, algorithms based on random drawings from a distribution $\mathbb{P}$ are able to integrate any $\mathbb{P}$-integrable function. On the other hand, the error of quasi-Monte Carlo algorithms based on $n$ points and Gaussian quadrature formulas with $n$ nodes goes to 0 as long as $n \rightarrow \infty$ for Riemann integrable functions defined on a bounded interval (see [16], Definition on page 311 and Theorem 7.2.2). ${ }^{6}$

Hypothesis 3 is often more complex to deal with, since it involves Lipschitz approximations. As an example, suppose that the class of functions $\{g(\cdot, x), x \in \mathbf{X}\}$ is uniformly equicontinuous, that is for every $\varepsilon>0$, there exists $\delta>0$ such that for any $x \in \mathbf{X}$ and all $y, y^{\prime} \in \mathbf{Y}$ such that $d\left(y, y^{\prime}\right)<\delta$, we have $\left|g(y, x)-g\left(y^{\prime}, x\right)\right|<\varepsilon$. This implies that the Lipschitz approximation is continuous in $\mathbf{X}$ (this implies, indeed, that also the class $\left\{g^{k}(\cdot, x), x \in \mathbf{X}\right\}$ is uniformly equicontinuous). This hypothesis does not rule out discontinuities of $g$ arising for a fixed value of $x_{0}$ and for any $y$.

\footnotetext{
${ }^{6}$ Using an algorithm based on the probability integral transform (see e.g. [1]) and on its multivariate generalization (see [29]), the result can be extended to Lebesgue-Riemann integrable functions. In [27], the univariate version of this method is dealt with under the name of method of inversion.
} 


\section{An Application to ams Transformations}

The objective of this Section is to derive a functional form of the Birkhoff Ergodic Theorem for asymptotically mean stationary transformations.

Consider a probability space $(\Omega, \mathcal{A}, \mathbb{Q})$ and an $\mathcal{A}$-measurable transformation $T: \Omega \rightarrow \Omega$. $T$ is said to be measure-preserving if $\mathbb{Q}\left(T^{-1} A\right)=\mathbb{Q}(A)$ for all $A \in \mathcal{A}$. More precisely, we say that $T$ preserves the $\mathbb{Q}-$ measure. Equivalently, $\mathbb{Q}$ is said to be stationary with respect to $T$. The sets $A \in \mathcal{A}$ that satisfy $T^{-1} A=A$ are called invariant sets and constitute a sub- $\sigma-$ field $\mathcal{I}$ of $\mathcal{A}$. A random variable $X$ is $\mathcal{I}$-measurable if and only if $X(\omega)=X(T \omega)$ for all $\omega \in \Omega: X$ is said to be an invariant random variable. The measurable and measure-preserving transformation $T$ is said to be ergodic if $\mathbb{Q}(A)=0$ or 1 for all invariant sets $A$. Another equivalent formulation is also used: a sequence $X_{1}, X_{2}, \ldots$ is said to be stationary if the random vectors $\left(X_{1}, \ldots, X_{n}\right)$ and $\left(X_{k+1}, \ldots, X_{n+k}\right)$ have the same distribution for all integers $n, k \geq 1$. Any stationary sequence $X_{1}, X_{2}, \ldots$ can almost surely be rewritten using a measurable and measure-preserving transformation $T$ as $X_{t}(\omega)=X_{0}\left(T^{t} \omega\right)$ (see e.g. Proposition 6.11 in [6]). The transformation $T: \Omega \rightarrow \Omega$ on $(\Omega, \mathcal{A}, \mathbb{Q})$ is said to be asymptotically mean stationary (ams) if the sequence $\frac{1}{n} \sum_{j=0}^{n-1} \mathbb{Q}\left(T^{-j} A\right)$ is convergent for all $A \in \mathcal{A}$ (see [13]). It is known from the Vitali-Hahn-Saks Theorem that $\lim _{n \rightarrow \infty} \frac{1}{n} \sum_{j=0}^{n-1} \mathbb{Q} T^{-j}$ is a probability measure that we indicate as $\mathbb{P}$ and call asymptotic mean of $\mathbb{Q}$. The interest of ams transformations lies in the fact that asymptotic mean stationarity is a necessary and sufficient condition for a Birkhoff Ergodic Theorem to hold (see Theorem 2 for the Birkhoff Ergodic Theorem for ams processes, and $[13,8]$ for the necessary and sufficient conditions). Moreover, it is possible to show that many processes that appear in Applied Probability (in particular Queueing Theory, see [30, 31, 28, 21], Information Theory, see $[13,11,17]$, etc.) are ams and that many real situations give rise to data that are well modelled by ams processes. In particular, the class of asymptotically mean stationary processes includes many subclasses of processes that are relevant for applications, such as stationary processes, cyclostationary processes (i.e. processes with seasonal variations that are stationary in a certain sense, see [5]), Markov processes with an invariant probability measure and even some dynamical systems. Therefore the following result is particularly suitable for approximations of stochastic programs using real data.

The following Theorem (see [8]) will be used to derive the main result of this Section.

Theorem 2. Let $(\Omega, \mathcal{A})$ be a measurable space, $T: \Omega \rightarrow \Omega$ be a measurable transformation and $X$ an extended real-valued positive random variable defined on $(\Omega, \mathcal{A})$. In addition, suppose that $\mathbb{Q}$ is an ams measure with respect to $T$ on $(\Omega, \mathcal{A})$ with stationary mean $\mathbb{P}$. Then, for $\mathbb{Q}-($ and $\mathbb{P}-)$ almost every $\omega \in \Omega$, one has 


$$
\lim _{n \rightarrow \infty} \frac{1}{n} \sum_{i=0}^{n-1} X\left(T^{i} \omega\right)=\mathbb{E}(X \mid \mathcal{I})(\omega),
$$

where each side can be equal to $+\infty$ or $-\infty$, and where $\mathbb{E}(X \mid \mathcal{I})$ denotes the conditional expectation taken on $(\Omega, \mathcal{A}, \mathbb{P})$.

We introduce the following hypothesis that will be useful in the proof of Corollary 1.

Hypothesis 2': $\mathbb{Q}$ is an asymptotically mean stationary probability measure defined on the measurable space $(\mathbf{Y}, \mathcal{B}(\mathbf{Y}))$ with stationary mean $\mathbb{P} ; \mathbb{P}$ is an ergodic probability measure. $\left(Y_{i}(\omega)\right)_{i=1, \ldots, n}=\left(Y_{0}\left(T^{i} \omega\right)\right)_{i=1, \ldots, n}$ is a realization of length $n$ from the probability measure $\mathbb{Q} . \mathbb{P}_{n}$ is a sequence of transition probabilities defined by

$$
\mathbb{P}_{n}(\omega, A)=\frac{1}{n} \sum_{i=1}^{n} \delta_{Y_{i}(\omega)}(A)=\frac{1}{n} \sum_{i=1}^{n} \delta_{Y_{0}\left(T^{i} \omega\right)}(A)
$$

for each $n \geq 1$.

Corollary 1. Let $(\mathbf{X}, d)$ be a metrizable separable space endowed with its Borel $\sigma$-algebra $\mathcal{B}(\mathbf{X}),(\mathbf{Y}, \rho)$ a metric space endowed with its Borel $\sigma$-algebra $\mathcal{B}(\mathbf{Y})$ and $g$ be a normal integrand on $\mathbf{Y} \times \mathbf{X}$ with values in $\overline{\mathbb{R}}$ respecting Hypothesis 1. Then, if Hypothesis 2' holds, there exists a $\mathbb{P}$ - and $\mathbb{Q}$-negligible subset $N$ of $\Omega$ such that, for every $x \in \mathbf{X}$ and $\omega \in \Omega \backslash N$, one has:

$$
\mathbb{E} g(Y, x)=\text { epi- } \lim _{n \rightarrow \infty} \frac{1}{n} \sum_{i=0}^{n-1} g\left(Y_{i}(\omega), x\right),
$$

where $\mathbb{E}$ denotes the expectation under the probability measure $\mathbb{P}$.

\section{Proofs}

Proof of Theorem 1. For any $n \geq 1$, define on $\Omega \times \mathbf{X}$ the function

$$
h_{n}(\omega, x) \triangleq\left[\mathbb{E}_{n} g(Y, x)\right](\omega) .
$$

To show (8), it is enough to prove the following two inequalities:

$$
\begin{aligned}
\text { epi- } \liminf _{n \rightarrow \infty} h_{n}(\omega, x) & \geq \mathbb{E} g(Y, x), & & \forall \omega \in \Omega \backslash N_{1}, \forall x \in \mathbf{X}, \\
\text { epi- } \limsup _{n \rightarrow \infty} h_{n}(\omega, x) & \leq \mathbb{E} g(Y, x), & & \forall \omega \in \Omega \backslash N_{2}, \forall x \in \mathbf{X},
\end{aligned}
$$

where $N_{1}$ and $N_{2}$ are some negligible subsets of $\Omega$ that will be specified in the following. Recall that, for any $\omega \in \Omega$ and for any fixed $k \geq 1$, the Lipschitz approximation of order $k$ of $h_{n}(\omega, \cdot)$ is defined by: 


$$
h_{n}^{k}(\omega, x) \triangleq \inf _{x^{\prime} \in X}\left\{h_{n}\left(\omega, x^{\prime}\right)+k \cdot d\left(x, x^{\prime}\right)\right\} \quad \forall x \in \mathbf{X} .
$$

Now, using the super-additivity of the infimum operation, we easily obtain:

$$
h_{n}^{k}(\omega, x) \geq\left[\mathbb{E}_{n} g^{k}(Y, x)\right](\omega) .
$$

An appeal to Proposition 4.4 in [15] shows that $g^{k}$ and $h_{n}^{k}$ are $\widehat{\mathcal{A}} \otimes \mathcal{B}(\mathbf{X})-$ measurable. Consequently, for any $x \in \mathbf{X}^{\star}$ and $k \geq 1$, we can apply Hypothesis 3 to $\left(\mathbb{E}_{n}\left[g^{k}(Y, x)\right]\right)_{n \geq 1}$. This proves the existence of a negligible subset $N_{1}(x, k)$ such that, for any $\omega \in \Omega \backslash N_{1}(x, k)$,

$$
\liminf _{n} h_{n}^{k}(\omega, x) \geq\left[\mathbb{E} g^{k}(Y, x)\right](\omega)
$$

Set $N_{1} \triangleq \bigcup_{x \in \mathbf{X}^{\star}} \bigcup_{k \geq 1} N_{1}(x, k)$, where we recall that $\mathbf{X}^{\star}$ is a dense countable subset of $\mathbf{X}$. Inequality (12) is valid for $\omega \in \Omega \backslash N_{1}, k \geq 1$ and $x \in \mathbf{X}^{\star}$; moreover, it remains valid for any $x \in \mathbf{X}$ because each side of (12) defines a Lipschitz function of $x$, with Lipschitz constant $k$. Then, taking the supremum, with respect to $k$, in both sides of (12) and using formula (20) together with the monotone convergence theorem, we obtain (9).

To prove (10), it is useful to put, for any $x \in \mathbf{X}, \phi(x)=\mathbb{E} g(Y, x)$ and, for any $k>1$ and $x \in \mathbf{X}, \phi^{k}(x)=\inf \left\{\phi\left(x^{\prime}\right)+k d\left(x, x^{\prime}\right): x^{\prime} \in \mathbf{X}\right\}$. First, observe that, due to the properness of $\phi, \phi^{k}$ is finite on $\mathbf{X}$. Further, for any $x \in \mathbf{X}^{\star}, p \geq 1$ and $k \geq 1$, one can find $x^{\prime}=x^{\prime}(x, p, k) \in \mathbf{X}$ such that $\phi\left(x^{\prime}\right)+k d\left(\bar{x}, x^{\prime}\right) \leq \phi^{k}(x)+\frac{1}{p}$. Hence, for each $x \in \mathbf{X}^{\star}$ and $k \geq 1$, the following equality holds true:

$$
\phi^{k}(x)=\inf \left\{\phi\left(x^{\prime}(x, p, k)\right)+k d\left(x, x^{\prime}(x, p, k)\right): p \geq 1\right\} .
$$

Further, applying Hypothesis 4 to the sequence $\left(\mathbb{E}_{n}\left[g\left(Y, x^{\prime}(x, p, k)\right)\right]\right)_{n>1}$, we can see that, for every $x \in \mathbf{X}^{\star}, k \geq 1$ and $p \geq 1$, there exists a negligible subset $N_{2}(x, p, k)$ such that, for every $\omega \in \Omega \backslash N_{2}(x, p, k)$,

$$
\limsup _{n}\left[\mathbb{E}_{n} g\left(Y, x^{\prime}(x, p, k)\right)\right](\omega) \leq \phi\left(x^{\prime}(x, p, k)\right) .
$$

Put $N_{2} \triangleq \bigcup_{x \in \mathbf{X}^{\star}} \bigcup_{p \geq 1} \bigcup_{k \geq 1} N_{2}(x, p, k)$ and consider $\omega \in \Omega \backslash N_{2}$. For any $x \in \mathbf{X}^{\star}$ and $k \geq 1$, we have:

$$
\limsup _{n} h_{n}^{k}(\omega, x) \leq \inf _{x^{\prime} \in \mathbf{X}} \limsup _{n \rightarrow \infty}\left[h_{n}\left(\omega, x^{\prime}\right)+k d\left(x, x^{\prime}\right)\right] .
$$

Restricting the infimum to the subset $\left\{x^{\prime}(x, p, k): p \geq 1\right\}$ and using (14) and (13), we obtain

$$
\limsup _{n} h_{n}^{k}(\omega, x) \leq \inf _{p \geq 1}\left[\phi\left(x^{\prime}(x, p, k), \omega\right)+k d\left(x, x^{\prime}(x, p, k)\right)\right]=\phi^{k}(x) .
$$

So, we have proved, for each $k \geq 1$ and $\omega \in \Omega \backslash N_{2}$, 


$$
\limsup _{n} h_{n}^{k}(\omega, x) \leq \phi^{k}(x) \quad \forall x \in \mathbf{X}^{\star} .
$$

Then, invoking once more the Lipschitz property, we conclude that (15) remains valid for all $x \in \mathbf{X}$. Finally, taking the supremum on $k$ in both sides of (15) and using (21), we get (10).

Proof of Corollary 1. Hypotheses 3 and 4 of Theorem 1 holds except on $\mathbb{P}$-negligible sets because of Theorem 2 ; this implies that $\mathbb{P}(N)=0$. Moreover, since $\mathbb{P}$ is ergodic, $\left[\mathbb{E}_{n}(g(Y, x) \mid \mathcal{I})\right](\omega)=\left[\mathbb{E}_{n} g(Y, x)\right](\omega)$. The fact that $\mathbb{P}(N)=\mathbb{Q}(N)=0$ derives from the properties of ams measures (see $[13])$.

\section{A Some Results on Epi-convergence}

We begin by a sequential characterization of epi-convergence (Proposition 1.14 in $[3])$.

Proposition 1. A sequence of functions $\left(\phi_{n}\right)_{n \in \mathbb{N}}$ from $E$ into the extended reals epi-converges to a function $\phi$ at $x \in E$, if and only if the following two properties (i) and (ii) hold:

(i) For each sequence $\left(x_{n}\right)$ converging to $x \in E$ one has

$$
\phi(x) \leq \liminf _{n \rightarrow \infty} \phi_{n}\left(x_{n}\right) .
$$

(ii) There exists a sequence $\left(x_{n}\right)$ in $E$ converging to $x$ and such that

$$
\phi(x) \geq \limsup _{n \rightarrow \infty} \phi_{n}\left(x_{n}\right) .
$$

Remark 2. Properties (i) and (ii) are equivalent to (i) and (ii') where:

(ii') There exists a sequence $\left(x_{n}\right)$ converging to $x$ such that $\phi(x)=\lim _{n \rightarrow \infty} \phi_{n}\left(x_{n}\right)$.

The variational properties of epi-convergence play a crucial role in the present paper. For this purpose, some notations are useful. For any extended realvalued function $\phi$ defined on $E$, we define the set of (exact) minimizers of $\phi$ on $E$ by setting

$$
\operatorname{Arg} \min (\phi) \triangleq\left\{x \in E: \phi(x)=\inf _{y \in E} \phi(y)\right\} .
$$

More generally, for a function $\phi$ such that the infimum on $E$ is different from $-\infty$, we define, for any $\alpha \geq 0$, the set of $\alpha$-approximate minimizers by

$$
\alpha-\operatorname{Arg} \min (\phi) \triangleq\left\{x \in E: \phi(x) \leq \inf _{y \in E} \phi(y)+\alpha\right\} .
$$

Whenever $\alpha>0$, the set of $\alpha$-approximate minimizers is non-empty (unlike the set of exact minimizers which is obtained for $\alpha=0$ ). The variational properties of epi-convergence that we need are stated in the following result (see Corollary 2.10 in [3]). 
Proposition 2. The following two results hold:

(i) Assume that $\left(\phi_{n}\right)$ is epi-convergent to $\phi$, that is $\phi=e p i-\lim _{n \rightarrow \infty} \phi_{n}$. Then, the following inequality holds

$$
\inf _{x \in E} \phi(x) \geq \limsup _{n \rightarrow \infty} \inf _{x \in E} \phi_{n}(x) .
$$

(ii) Let $\left(\alpha_{n}\right)_{n \in \mathbb{N}}$ be a sequence of positive reals converging to 0 . For any $n \geq 1$, let $x_{n}$ be an $\alpha_{n}$-approximate minimizer of $\phi_{n}$. If the sequence $\left(x_{n}\right)$ admits a subsequence converging to some $x \in E$, then $x$ belongs to $\operatorname{Arg} \min (\phi)$ and (16) becomes

$$
\min _{x \in E} \phi(x)=\limsup _{n \rightarrow \infty} \inf _{x \in E} \phi_{n}(x) .
$$

Now we introduce some further properties of epi-convergence, and in particular its relation with uniform convergence. We recall that the lower (resp. upper) hypo-limits, as well as the hypo-convergence of a sequence $\left(\phi_{n}\right)$, can be obtained from the corresponding epigraphical concepts in a symmetric way. Indeed, the sequence $\left(\phi_{n}\right)$ hypo-converges to $\phi$ iff $\left(-\phi_{n}\right)$ epi-converges to $-\phi$. In order to present the relation between epi-convergence, hypo-convergence and uniform convergence, we remark that replacing $\left(\phi_{n}\right)$ with $\left(-\phi_{n}\right)$ and $\phi$ with $-\phi$ in the statement of Proposition 1, we get similar characterizations of hypo-convergence. Consequently, a sequence $\left(\phi_{n}\right)$ is both epi- and hypoconvergent to $\phi$ if and only if the following property holds:

$$
\forall x \in E, \forall\left(x_{n}\right) \rightarrow x, \quad \phi(x)=\lim _{n \rightarrow \infty} \phi_{n}\left(x_{n}\right) .
$$

The following simple result shows the connection with uniform convergence.

Proposition 3. If $\phi$ and $\left(\phi_{n}\right)$ satisfy (17), then $\phi$ is continuous and $\left(\phi_{n}\right)$ converges uniformly to $\phi$ on all compact sets.

Proof. First observe that Remark 2 shows that $\left(\phi_{n}\right)$ is both epi- and hypoconvergent to $\phi$. Thus $\phi$ is both lower and upper semi-continuous, hence continuous on $E$. Further consider a compact subset $K$ of $E$ and suppose that $\left(\phi_{n}\right)$ does not converge uniformly to $\phi$ on $K$. It is therefore possible to find $\alpha>0$ and a subsequence $\left(\phi_{m}\right)$ of $\left(\phi_{n}\right)$ satisfying:

$$
\left\|\phi_{m}-\phi\right\|_{\mathrm{u}, \mathrm{K}}=\sup _{x \in K}\left|\phi_{m}(x)-\phi(x)\right| \geq \alpha>0 \quad \text { for all } m \geq 1 .
$$

But for all $m \geq 1$, there exists $x_{m} \in K$ such that

$$
\left|\phi_{m}\left(x_{m}\right)-\phi\left(x_{m}\right)\right| \geq\left\|\phi_{m}-\phi\right\|_{\mathrm{u}, \mathrm{K}}-\frac{1}{m} .
$$

Moreover, by extracting a subsequence (denoted similarly) converging to some $x \in K$, we have 


$$
\left|\phi_{m}\left(x_{m}\right)-\phi(x)\right| \geq\left|\phi_{m}\left(x_{m}\right)-\phi\left(x_{m}\right)\right|-\left|\phi\left(x_{m}\right)-\phi(x)\right| .
$$

From (18) and (19), we get

$$
\left|\phi_{m}\left(x_{m}\right)-\phi(x)\right| \geq\left\|\phi_{m}-\phi\right\|_{\mathrm{u}, \mathrm{K}}-\frac{1}{m}-\left|\phi\left(x_{m}\right)-\phi(x)\right| .
$$

Thus $\liminf _{m \rightarrow \infty}\left|\phi_{m}\left(x_{m}\right)-\phi(x)\right| \geq \alpha>0$, which contradicts property (17).

Epi-convergence can be conveniently characterized by means of the Lipschitz approximations (see e.g. Proposition 3.4 of [15]). Given a Isc function $\phi: E \rightarrow \overline{\mathbb{R}}$ and an integer $k \geq 1$, the Lipschitz approximation of order $k$ of $\phi$ is defined by:

$$
\phi^{k}(x) \triangleq \inf _{y \in E}\{\phi(y)+k \cdot d(x, y)\}, \quad k \geq 1 .
$$

Its main properties are listed in the following Proposition (see [15], Proposition $3.3)$.

Proposition 4. Let $\phi: E \rightarrow \overline{\mathbb{R}}$ be a Isc function non identically equal to $+\infty$. Suppose that there exists $a>0, b \in \mathbb{R}$ and $x_{0} \in E$ such that, for all $x \in E, \phi(x)+a \cdot d\left(x, x_{0}\right)+b \geq 0$. Then:

(i) $\forall k>a$ and $\forall x \in E, \quad \phi^{k}(x)+a \cdot d\left(x, x_{0}\right)+b \geq 0$,

(ii) $\forall k \geq 1, \phi^{k}<+\infty$ and $\phi^{k}$ is Lipschitz of constant $k$,

(iii) $\forall x \in E$, the sequence $\left(\phi^{k}(x)\right)_{k \geq 1}$ is increasing and $\phi(x)=\sup _{k \geq 1} \phi^{k}(x)$.

The role of Lipschitz approximations in epi-convergence is explained in the following result (see [15], Proposition 3.4).

Proposition 5. Let $\left(\phi_{n}\right)$ be a sequence of functions from $E$ to $\overline{\mathbb{R}}$ satisfying: there exist $a>0, b \in \mathbb{R}$ and $x_{0} \in E$ such that, for every $n \geq 1$ and $x \in E$, $\phi_{n}(x)+a \cdot d\left(x, x_{0}\right)+b \geq 0$. Then for all $x \in E$ :

$$
\begin{aligned}
\text { epi- } \liminf \phi_{n}(x) & =\sup _{k \geq 1} \liminf _{n \rightarrow \infty} \phi_{n}^{k}(x) \\
\text { epi- } \lim \sup \phi_{n}(x) & =\sup _{k \geq 1} \limsup _{n \rightarrow \infty} \phi_{n}^{k}(x)
\end{aligned}
$$

Acknowledgement. We would like to thank the editor D. Talay and a referee for providing useful comments and corrections.

\section{References}

1. J.E. Angus. The probability integral transform and related results. SIAM Rev., 36(4):652-654, 1994. 
2. Z. Artstein and R.J.-B. Wets. Consistency of minimizers and the SLLN for stochastic programs. J. Convex Anal., 2(1-2):1-17, 1995.

3. H. Attouch. Variational convergence for functions and operators. Applicable Mathematics Series. Pitman (Advanced Publishing Program), Boston, MA, 1984.

4. J.R. Birge and F. Louveaux. Introduction to stochastic programming. Springer Series in Operations Research. Springer-Verlag, New York, 1997.

5. R.A. Boyles and W.A. Gardner. Cycloergodic properties of discrete-parameter nonstationary stochastic processes. IEEE Trans. Inform. Theory, 29(1):105114, 1983.

6. L. Breiman. Probability, volume 7 of Classics in Applied Mathematics. Society for Industrial and Applied Mathematics (SIAM), Philadelphia, PA, 1992.

7. C. Choirat, C. Hess, and R. Seri. A functional version of the Birkhoff ergodic theorem for a normal integrand: a variational approach. Ann. Probab., 31(1):6392, 2003.

8. C. Choirat, C. Hess, and R. Seri. Ergodic theorems for extended real-valued random variables. Working paper, 2004.

9. G. Dal Maso. An introduction to $\Gamma$-convergence. Progress in Nonlinear Differential Equations and their Applications, 8. Birkhäuser Boston Inc., Boston, MA, 1993.

10. K.-T. Fang and Y. Wang. Number-theoretic methods in statistics, volume 51 of Monographs on Statistics and Applied Probability. Chapman \& Hall, London, 1994.

11. R.J. Fontana, R.M. Gray, and J.C. Kieffer. Asymptotically mean stationary channels. IEEE Trans. Inform. Theory, 27(3):308-316, 1981.

12. I.I. Gikhman and A.V. Skorokhod. Introduction to the theory of random processes. Translated from the Russian by Scripta Technica, Inc. W. B. Saunders Co., Philadelphia, Pa., 1969.

13. R.M. Gray and J.C. Kieffer. Asymptotically mean stationary measures. Ann. Probab., 8(5):962-973, 1980.

14. C. Hess. Epi-convergence of sequences of normal integrands and strong consistency of the maximum likelihood estimator. Cahiers de Mathématiques de la Décision, No. 9121, Université Paris Dauphine, 1991.

15. C. Hess. Epi-convergence of sequences of normal integrands and strong consistency of the maximum likelihood estimator. Ann. Statist., 24(3):1298-1315, 1996.

16. K.L. Judd. Numerical methods in economics. MIT Press, Cambridge, MA, 1998.

17. J.C. Kieffer and M. Rahe. Markov channels are asymptotically mean stationary. SIAM J. Math. Anal., 12(3):293-305, 1981.

18. M. Koivu. Variance reduction in sample approximations of stochastic programs. Working Paper, 2004.

19. L.A. Korf and R.J.-B. Wets. Random-lsc functions: an ergodic theorem. Math. Oper. Res., 26(2):421-445, 2001.

20. J.-J. Liang, K.-T. Fang, F.J. Hickernell, and R. Li. Testing multivariate uniformity and its applications. Math. Comp., 70(233):337-355, 2001.

21. T. Nakatsuka. Absorbing process in recursive stochastic equations. J. Appl. Probab., 35(2):418-426, 1998.

22. J. Neveu. Bases mathématiques du calcul des probabilités. Masson et Cie, Éditeurs, Paris, 1964. 
23. A.B. Owen. Monte Carlo variance of scrambled net quadrature. SIAM J. Numer. Anal., 34(5):1884-1910, 1997.

24. A.B. Owen. Scrambled net variance for integrals of smooth functions. Ann. Statist., 25(4):1541-1562, 1997.

25. T. Pennanen. Epi-convergent discretizations of multistage stochastic programs. Stochastic Programming E-Print Series, Number 2004-03, 2004.

26. T. Pennanen and M. Koivu. Integration quadratures in discretizations of stochastic programs. Stochastic Programming E-Print Series, Number 2002-11, 2002.

27. T. Pennanen and M. Koivu. Epi-convergent discretizations of stochastic programs via integration quadratures. Stochastic Programming E-Print Series, Number 2003-16, 2003.

28. T. Rolski. Queues with nonstationary inputs. Queueing Systems Theory Appl., 5(1-3):113-129, 1989.

29. M. Rosenblatt. Remarks on a multivariate transformation. Ann. Math. Statistics, 23:470-472, 1952.

30. W. Szczotka. Stationary representation of queues. I. Adv. in Appl. Probab., 18(3):815-848, 1986.

31. W. Szczotka. Stationary representation of queues. II. Adv. in Appl. Probab., 18(3):849-859, 1986.

32. M. Valadier. Conditional expectation and ergodic theorem for a positive integrand. J. Nonlinear Convex Anal., 1(3):233-244, 2000.

33. M. Valadier. What differentiates stationary stochastic processes from ergodic ones: a survey. Sūrikaisekikenkyūsho Kōkyūroku, (1215):33-52, 2001. 